\title{
Preface
}

\section{Sleep Disturbances and \\ Disorders: A Poorly \\ Recognized Accident Risk}

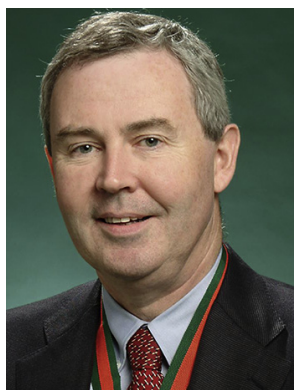

Walter T. McNicholas, MD

Editor
Speed and alcohol consumption are widely recognized contributory factors to motor vehicle accidents (MVA) and are almost universally regulated throughout the world by speed limits and blood/urine/breath alcohol levels while driving. In recent decades, there has been growing recognition that sleep disturbances and disorders with consequent sleepiness during the waking hours also represent important contributing factors to driving accident risk. ${ }^{1}$ However, this risk is difficult to quantify by objective measures such as are employed for speed and alcohol, which inevitably result in sleepiness being a less well-documented contributing factor to MVA. Nonetheless, driving while sleepy confers a similar increased accident risk as driving with a blood alcohol level above the legal limit for most countries. Various reports estimate that sleepiness represents a major contributing factor in about $20 \%$ of serious MVA and is particularly likely to be a major factor in MVA on major highways where monotonous driving at relatively high speed is common. ${ }^{2}$

Fatigue and sleepiness because of sleep dysfunction are most common in circumstances of poor sleep hygiene where the affected individual spends too little time in bed and/or experiences disturbed sleep due to poor lifestyle habits. Specific medical disorders may also contribute to sleep disturbance, the most important of which is obstructive sleep apnea (OSA).
OSA is widely reported to increase MVA risk 2- to 3-fold, but this increased risk is removed by effective treatment, which underlines the practical importance of recognition and treatment. ${ }^{3}$ Indeed, several jurisdictions have introduced regulations that preclude untreated patients with moderate or severe OSA associated with sleepiness from driving unless effectively treated. ${ }^{4,5}$

The present issue of Sleep Medicine Clinics is devoted to the topic of Sleep and Driving and provides a comprehensive review of all aspects of this relationship. Topics discussed include the impact on driving safety of sleep restriction, sleep hygiene, and shift work, in addition to the economic burden of sleepy drivers. The role of OSA in MVA risk is extensively discussed, including the role of screening, effective treatment, and the role of government regulation. The assessment of sleepiness in drivers is an important practical topic, as current limitations in techniques to assess sleepiness limit the ability to objectively measure this factor, and future possibilities, especially technology related, are reviewed. Vehicle and highway adaptations to compensate for sleepy drivers are of increasing importance in promoting driving safety. While these measures are directed at all drivers, they have special relevance to the sleepy driver. The role of the employer in screening for sleep disorders among professional drivers is also discussed as there is growing evidence that targeted screening of truck drivers for disorders such as 
OSA confers financial benefits to the industry as well as improved highway safety. ${ }^{6}$

Walter T. McNicholas, MD School of Medicine

University College Dublin Department of Respiratory and Sleep Medicine

St. Vincent's Hospital Group Merrion Road, Dublin 4, Ireland

First Affiliated Hospital of Guangzhou Medical University Guangzhou, China

E-mail address: walter.mcnicholas@ucd.ie

\section{REFERENCES}

1. Bioulac S, Franchi J-AM, Arnaud M, et al. Risk of motor vehicle accidents related to sleepiness at the wheel: a systematic review and meta-analysis. Sleep 2018;41(7).

2. Czeisler CA, Wickwire EM, Barger LK, et al. Sleepdeprived motor vehicle operators are unfit to drive: a multidisciplinary expert consensus statement on drowsy driving. Sleep Health 2016;2(2):94-9.

3. Tregear S, Reston J, Schoelles K, et al. Continuous positive airway pressure reduces risk of motor vehicle crash among drivers with obstructive sleep apnea: systematic review and meta-analysis. Sleep 2010; 33(10): 1373-80.

4. Bonsignore MR, Randerath W, Riha R, et al. New rules on driver licensing for patients with obstructive sleep apnea: European Union Directive 2014/85/EU. J Sleep Res 2016;25(1):3-4.

5. McNicholas WT, Rodenstein D. Sleep apnoea and driving risk: the need for regulation. Eur Respir Rev 2015;24(138):602-6.

6. Burks SV, Anderson JE, Bombyk M, et al. Nonadherence with employer-mandated sleep apnea treatment and increased risk of serious truck crashes. Sleep 2016;39(5):967-75. 